\title{
PRODUÇÃO CIENTÍFICA BRASILEIRA NAS 40 REVISTAS DE PSIQUIATRIA COM MAIOR FATOR DE IMPACTO NO ANO DE 2006
}

Felipe filardi da Rocha, Thiago fuscaldi, Viviam Castro, William do Carmo, Débora amaral, humberto Correa*

Trabalho realizado no Departamento de Saúde Mental - Faculdade de Medicina da UFMG -Belo Horizonte-MG

\section{*Correspondência}

Faculdade de Medicina UFMG

Departamento de Saúde Mental

Av. Alfredo Balena, 190

Belo Horizonte - MG

Cep 30130-100

\section{RESUMO}

Oвjetivo. Avaliar a produção científica brasileira, no ano de 2006, nas 40 revistas de psiquiatria com maior fator de impacto (FI) segundo o Journal of Citation Report (JCR), 2006.

Métodos. Do total de 94 periódicos, selecionamos todos os artigos com Fl acima da média $(n=40)$. Avaliamos, entre estes artigos, quantos apresentavam pelo menos um autor brasileiro, quantos eram compostos por equipe exclusivamente brasileira ou tinham pelo menos o primeiro autor e/ou 0 autor sênior filiado à instituição nacional. Avaliamos também a respectiva distribuição das publicaçõoes por estados, de acordo com a instituiç̧ão de filiação do(s) autor(es).

Resultados. Entre 7.996 artigos avaliados, 96 (1,2\%) tinham pelo menos um autor com afliação em instituição brasileira e oito tinham autor sênior brasileiro, sendo excluídas as 59 publicações compostas exclusivamente por pesquisadores brasileiros. Ao se determinar a distribuição, por estados, da instituição de pesquisa na qual está(ão) vinculado(s) o(s) autor(res) brasileiro(s), São Paulo, com 46 publicações (47,91\%), ficou na primeira posiçã̃o, com predominância absoluta do eixo Sudeste-Sul $(98,95 \%)$.

Conclusão. Apesar das publicações brasileiras em psiquiatria terem apresentado aparente crescimento quantitativo e qualitativo, a produção científica da área é ainda pequena e altamente concentrada em alguns poucos estados.

Unitermos: Análise bibliométrica. Periódicos. Psiquiatria. Pesquisa. Fator de impacto.

\section{INTRODUÇÃO}

Acessando o site http://esi3.isiknowledge.com/rankdatapage.cgi, em $1^{\circ}$ de fevereiro de 2006, obtivemos o JCR de 2006, em que constam 94 periódicos de psiquiatria com Fl variando de 12,64 até $0,00(2.43 \pm 2,04)$. Para nosso estudo, selecionamos todos os periódicos com Fl acima da média, totalizando 40 periódicos. Consideramos em nossa análise todos os tipos de artigo, exceto os comentários sobre livros e erratas. Entre estes artigos, avaliamos inicialmente aqueles em que pelo menos um autor tem afiliação a uma instituição brasileira.

A seguir, analisamos os artigos em que pelo menos o primeiro autor e/ou o autor sênior têm afiliação à instituição brasileira, e artigos em que todos os autores apresentam afiliação à instituiç̧ão brasileira, ou seja, artigos produzidos por uma equipe trabalhando exclusivamente no Brasil. Avaliamos, entre os artigos selecionados, a respectiva distribuição das publicações por estados, de acordo com a instituição de pesquisa na qual o pesquisador está afiliado. A análise estatística foi realizada com o programa SPSS v. 14 para Windows.

\section{Métodos}

Os 40 periódicos de psiquiatria indexados no $|\mathrm{S}|$ e citados no JCR de 2006 com Fl acima da média geral estão listados na Tabela I. Para cada periódico obtivemos o número de artigos publicados em 2006, o que constitui um total de 7.996 artigos. Destes, 96
$(1,2 \%)$ apresentavam pelo menos um autor afiliado a uma instituição brasileira. Artigos produzidos por equipe exclusivamente brasileira representaram 59 publicações, mais da metade dos artigos publicados com pelo menos um autor brasileiro $(61,45 \%)$ (Tabela I).

Ao serem analisados os 96 artigos e determinada a distribuição por Estados da instituição de pesquisa a qual está(ão) vinculado(s) o(s) autor(res) brasileiro(s), observamos que o Estado de São Paulo, com 46 publicações (47,91\%), ficou na primeira posição, seguido pelo Rio Grande do Sul, com 27 publicações (28,12\%), e Rio de Janeiro, com dez (10,41\%). O eixo Sudeste-Sul, com 98,95\% dos artigos, ressalta a intensa concentração da produção científica em psiquiatria em nosso país (Tabela 2).

\section{Dıscussão}

Recentemente, diversos estudos vêm tentando caracterizar quantitativamente e qualitativamente a produção científica brasileira, principalmente no cenário internacional ${ }^{1-8}$. A produção científica brasileira, de forma geral, apresentou um crescimento de quatro vezes, desde a década 1980, no que se refere ao total de artigos publicados. Hoje nos encontramos na $17^{\text {a }}$ colocação mundial e em primeiro lugar na América Latina, com uma produção, em 2004, que correspondia a aproximadamente $1,7 \%$ da produção científica mundial2,9.

Dentro deste contexto, as áreas da saúde e ciências biológicas são as principais contribuintes, constituindo quase $45 \%$ dos artigos 


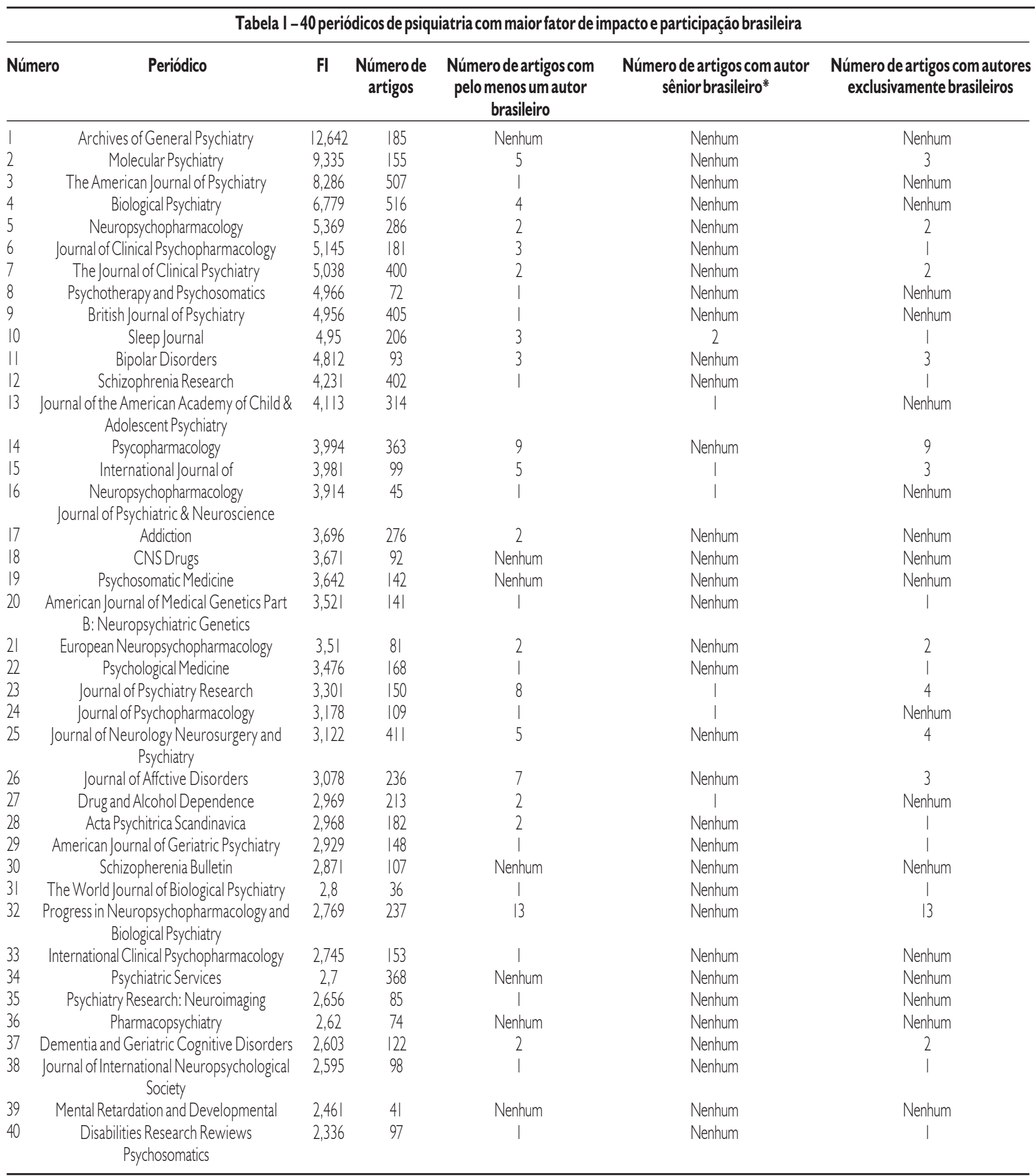

* Excluindo-se aqueles com autores exclusivamente brasileiros 


\begin{tabular}{|c|c|c|}
\hline Estados & $\begin{array}{l}\text { Númerode } \\
\text { publicações }\end{array}$ & $\begin{array}{l}\text { Número de programas de pós-graduação } \\
\text { strictu sensu nas áreas de psiquiatria, saúde } \\
\text { mental, neuropsiquiatria e psicobiologia }\end{array}$ \\
\hline São Paulo & 46 & 5 \\
\hline Rio Grande do Sul & 27 & I \\
\hline Rio de Janeiro & 10 & | \\
\hline Santa Catarina & 3 & 0 \\
\hline Minas Gerais & 4 & 0 \\
\hline Paraná & 5 & 0 \\
\hline Distrito Federal & I & 0 \\
\hline Total & 96 & 7 \\
\hline
\end{tabular}

brasileiros citados no ISI no período de 1998 a 2002. A área médica representa quase um quarto desta produção, e mesmo subáreas menos tradicionais, como a psiquiatria e a psicologia apresentaram, nos últimos anos, considerável crescimento ${ }^{2,10}$.

Há dois anos, Bressan, Gerolin e Mari avaliaram a produção científica nacional das áreas de psiquiatria, psicobiologia e saúde mental, no período compreendido entre 1998 e 2002, em nove programas nacionais de pós-graduação stricto sensu das áreas citadas. Estes autores concluíram que, segundo dados do $J C R$ de 2004, o Brasil é o $28^{\circ}$ país no ranking de citações e número de artigos indexados no ISI na área de psiquiatria/psicologia, tendo dobrado, no período, o número de publicações no $|S|^{5}$.

Recentemente, dados mostraram que, dentre os países da América Latina, o Brasil se destaca por ser o que possui maior número de citações por artigo no ISI nas áreas da psiquiatria e psicologia, enquanto o México se apresenta em primeiro lugar no quesito número de artigos. Outro dado interessante é que $60 \%$ dos artigos brasileiros foram publicados em periódicos com alto fator de impacto $(\mathrm{FI}>2)^{8}$

\section{Conclusão}

Ao analisarmos nossos dados, observamos que um número expressivo, apesar de pequeno, das produções científicas em periódicos psiquiátricos com $\mathrm{Fl}$ acima da média apresenta pelo menos um autor brasileiro, sendo que mais da metade foram produzidos por equipes exclusivamente brasileiras.

Um fato que merece destaque é uma certa homogeneidade da participação brasileira entre estes 40 periódicos averiguados. Poderia-se questionar que a presença nacional estaria concentrada entre os periódicos com os menores Fls. Porém, adotando-se o percentil 75 dos Fls $(3,17)$, teremos um total de 23 periódicos acima deste limite, totalizando 5.279 artigos publicados, sendo 58 $(1,09 \%)$ com pelo menos um autor brasileiro e 33 (56,89\%) com todos os autores pertencentes a uma instituição brasileira de pesquisa. Percebe-se que essas taxas são muito próximas das encontradas quando abrangemos todos os periódicos acima da média.

Além disso, gostaríamos de ressaltar que estes valores são

Tabela 3-Representatividade brasileira entre os principais periódicos de
psiquiatria nos anos de 2005 e 2006

semelhantes a um artigo publicado por nosso grupo anteriormente, quando avaliamos a participação brasileira entre os periódicos com Fl acima do percentil 75, no ano de 2005' (Galileu et al., 2006) (Tabela 3). Aparentemente, a contribuição nacional entre os periódicos psiquiátricos de maiores Fls tem se mostrado uma característica consistente. Porém, apenas acompanhando estes índices, poderemos ter informações mais conclusivas.

Apesar disso, as publicações brasileiras em psiquiatria representam uma fração ainda reduzida do percentual global em medicina (I,9\% no Brasil versus 6\% do percentual mundial). Com o aprimoramento da avaliaçãa dos programas de pós-graduação no País, tendo-se como critério essencial a relevância da produção intelectual aferida pela qualidade dos veículos cientíicos, espera-se, em pouco tempo, uma redução desta distância ${ }^{10}$. A produção científica brasileira nestas áreas, assim como em outras, concentrase nas regiões Sul e Sudeste, e são provenientes de um número restrito de centros de pesquisa que, por sua vez, possuem os principais e mais renomados programas de pós-graduação".

Recentemente, Zorzetto et al. (2006), ao avaliarem as 20 principais instituições brasileiras com maior número de artigos indexados pelo ISI durante o período de 1998 a 2003, observaram que estas são responsáveis por $78,7 \%$ da produção científica e que I5 se encontravam no eixo Sudeste-Sul (I2 na região Sudeste e três na região Sul). Mais especificamente, dentre estas 20 primeiras colocadas, nove (45\%) situavam-se no Estado de São Paulo².

Em nosso estudo, observamos dados que não contradizem esta situação, com $98,95 \%$ das publicações sendo procedentes do eixo Sudeste-Sul, e o Estado de São Paulo correspondendo a $47,91 \%$ do total de produções. Destaca-se o fato de sete dos nove (77,77\%) programas de pós-graduação strictu sensu nas áreas de psiquiatria, saúde mental, neuropsiquiatria e psicobiologia, estarem situados nesse eixo.

Muito já se discute a respeito da necessidade do investimento e aperfeiçoamento dos cursos de pós-graduação para a melhora quantitativa e qualitativa da produção científica brasileira. Estes cursos permitem, além da produção científica em si, a qualificação de profissionais que poderão desenvolver e coordenar linhas de pesquisa específicas, o que, por si só, já aumentará a quantidade de artigos produzidos.

Estima-se que o crescimento no número de doutores na última década tenha sido de aproximadamente $14,5 \%$ ao ano, com 
DA ROCHA FF ET AL.

número cinco vezes maior de alunos que receberam o título em 2001 do que em 1990. Neste ritmo, no final da década atual, o Brasil vai estar formando mais doutores do que países como o Japão e os Estados Unidos. As áreas da saúde e ciências biológicas são responsáveis por aproximadamente $25 \%$ desses títulos fornecidos $^{2}$.

Apesar deste crescimento, o cenário científico brasileiro não é adequado para acolher este número de pesquisadores, com baixos salários, exaustivas jornadas de trabalho e baixo investimento em infra-estrutura. Outro fato preocupante é a discrepância entre 0 avanço cientíico e os reais benefícios que os mesmos trazem para a assistência básica na saúde. No Brasil, é nítida a carência de políticas adequadas de saúde mental, além da falta de profissionais em centros de atenção primária da saúde.

Conflito de interesse: não há.

\section{SUMMARY}

\section{BRAZILIAN SCIENTIFIC PRODUCTION IN THE 40 PSYCHIATRIC JOURNALS WITH HIGH IMPACT FACTOR IN 2006}

OBIECTIVE. The Brazilian scientific production published in 2006 in the twenty psychiatric journals with high impact factor (IF) according to the Journal of Citation Report (JCR), 2006, was evaluated.

METHODS. All articles from 94 journals with an IF higher than the average $(n=40)$ were selected. We assessed the articles that had at least one author affiliated to a Brazilian institution as well as those with only Brazilian authors or a first/senior author belonging to a Brazilian institution. Secondly, the distribution of the author(s) by state was determined.

RESULTS. Among 7996 articles surveyed 96 (1.20\%) had at least one author from a Brazilian institution and in eight, the first senior author belonged to a Brazilian institution, not including the 59 publications that were written only by Brazilian authors. The distribution by state showed São Paulo as ranking first with 46 articles (47.91\%) and absolute predominance of the SouthSoutheast region (98.95\%).
CONCLUSION. Although, Brazilian publications on psychiatry had demonstrated apparent quantitative and qualitative growth, scientific production on the subject remains highly concentrated in a few states. [Rev Assoc Med Bras 2007; 53(6): 543-6]

KEY wORDS: Bibliometric analysis. Journals. Psychiatry. Research. Impact factor.

\section{REFERÊNCIAS}

I. Galileu D, Rocha FF, Nicolato R, Teixeira AL, Romano-Silva MA, Correa $\mathrm{H}$. Brazilian production in psychiatric journals with high impact factor in 2005. J Bras Psiquiatr. 2006;55:120-4.

2. Zorzetto R, Razzouk D, Dubugras MT, Gerolin J, Schor N, Guimaraes JA, et al.. The scientific production in health and biological sciences of the top 20 Brazilian universities. Braz J Med Biol Res. 2006;39:1513-20.

3. Garfield E. SCl Journal Citation Reports: a bibliometric analysis of science journals in the ISI database. Philadelphia (PA): Institute for Scientific Information; 1995.

4. Kieling C, Gonçalves RRF. Assessing the quality of a scientific journal: the case of Revista Brasileira de Psiquiatria. Rev Bras Psiquiatr. 2007;29:177-81.

5. Bressan RA, Gerolin J, Mari JJ. The modest but growing Brazilian presence in psychiatric, psychobiological and mental health research: assessment of the 1998-2002 period. Braz J Med Biol Res. 2005;5:649-59.

6. Da Rocha FF, Lage NV, De Sousa KCA, Correa H. Onde estão sendo publicados os estudos sobre suicídio no Brasil? Rev Bras Psiquiatr. 2007. [No prelo]

7. Guimarães J. Medical and biomedical research in Brazil. A comparison of Brazilian and international scientific performance. Ciênc Saúde Coletiva. 2004;9:307-27.

8. Razzouk D, Zorzetto R, Dubugras MTB, Gerolin J, Mari Jde J. Leading countries in mental health research in Latin America and the Caribbean. Rev Bras Psiquiatr. 2007;29:118-22.

9. King DA. The scientific impact of nations. Nature. 2004;430:3 I I-6.

10. Mari JJ. A crescente presença do Brasil no cenário científico internacional. Rev Bras Psiquiatr. 2002;24: 61-2.

I I. Velloso A, Lannes D, De Meis L. Concentration of science in Brazilian governmental universities. Scientometrics. 2004;61:207-20.

Artigo recebido: 08/08/07

Aceito para publicação: 19/09/07 